\title{
Moufang loops and generalized Lie-Cartan theorem ${ }^{1}$
}

Eugen PAAL

Tallinn University of Technology, Ehitajate tee 5, 19086 Tallinn, Estonia

E-mail: eugen.paal@ttu.ee

\begin{abstract}
Generalized Lie-Cartan theorem for linear birepresentations of an analytic Moufang loop is considered. The commutation relations of the generators of the birepresentation are found. In particular, the Lie algebra of the multiplication group of the birepresentation is explicitly given.
\end{abstract}

2000 MSC: 20N05, 17D10, 20G05

Dedicated to Maks A. Akivis on the occasion of his 85 th birthday and 65 years of scientific activity

\section{Introduction}

Continuous symmetries generated by the Lie transformation groups are widely exploited in modern mathematics and physics. Nevertheless, it may happen that the group theoretical methods are too rigid and one has to extend these beyond the Lie groups and algebras. From this point of view it is interesting to extend the group theoretical symmetry methods by using the Moufang loops and Mal'tsev algebras. The latter are known as minimal non-associative generalizations of the group and Lie algebra concepts, respectively.

In this paper, the generalized Lie-Cartan theorem for linear birepresentations of an analytic Moufang loop is considered. The commutation relations of the generators of the birepresentation were found. In particular, the Lie algebra of the multiplication group of the birepresentation is explicitly given.

Based on this theorem, various applications are possible. In particular, it was recently shown [6] how the Moufang-Noether current algebras may be constructed so that the corresponding Noether charge algebra turns out to be a birepresentation of the tangent Mal'tsev algebra of an analytic Moufang loop.

\section{Moufang loops}

A Moufang loop $[2,1,3]$ is a quasigroup $G$ with the unit element $e \in G$ and the Moufang identity

$$
(a g)(h a)=a(g h) a, \quad a, g, h \in G
$$

Here the multiplication is denoted by juxtaposition. In general, the multiplication need not be associative: $g h \cdot a \neq g \cdot h a$ for some triple of elements $a, g, h \in G$. The inverse element $g^{-1}$ of $g$ is defined by

$$
g g^{-1}=g^{-1} g=e
$$

\footnotetext{
${ }^{1}$ Presented at the International Conference "Loops '07", Prague, August 12-19, 2007.
} 


\section{Analytic Moufang loops and Mal'tsev algebras}

Following the concept of the Lie group, the notion of an analytic Moufang loop can be easily formulated.

A Moufang loop $G$ is said [4] to be analytic if $G$ is also a real analytic manifold and main operations - multiplication and inversion map $g \mapsto g^{-1}$ - are analytic mappings.

Let the local coordinates of $g$ from the vicinity of $e \in G$ be denoted by $g^{i}(i=1, \ldots, r \doteq$ $\operatorname{dim} G)$. The tangent space of $G$ at $e \in G$ is denoted by $T_{e}(G)$.

As in the case of the Lie groups, the structure constants $c_{j k}^{i}$ of an analytic Moufang loop are defined by

$$
\left.c_{j k}^{i} \doteq \frac{\partial^{2}\left(g h g^{-1} h^{-1}\right)^{i}}{\partial g^{j} \partial h^{k}}\right|_{g=h=e}=-c_{k j}^{i}, \quad i, j, k=1, \ldots, r
$$

For any $x, y \in T_{e}(G)$, their (tangent) product $[x, y] \in T_{e}(G)$ is defined in component form by

$$
[x, y]^{i} \doteq c_{j k}^{i} x^{j} y^{k}=-[y, x]^{i}, \quad i=1, \ldots, r
$$

The tangent space $T_{e}(G)$ being equipped with such an anti-commutative multiplication is called the tangent algebra of the analytic Moufang loop $G$. We shall use the notation $\Gamma \doteq\left\{T_{e}(G),[\cdot, \cdot]\right\}$ for the tangent algebra of $G$.

The tangent algebra of $G$ need not be a Lie algebra. There may exist a triple $x, y, z \in T_{e}(G)$ that does not satisfy the Jacobi identity:

$$
J(x, y, z) \doteq[x,[y, z]]+[y,[z, x]]+[z,[x, y]] \neq 0
$$

Instead, for all $x, y, z \in T_{e}(G)$ one has a more general Mal'tsev identity [4]

$$
[J(x, y, z), x]=J(x, y,[x, z])
$$

Anti-commutative algebras with this identity are called the Mal'tsev algebras.

\section{Birepresentation of a Moufang loop}

Consider a pair $(S, T)$ of the maps $g \mapsto S_{g}, g \mapsto T_{g}$ of a Moufang loop $G$ into $G L_{n}$. The pair $(S, T)$ is called [5] a (linear) birepresentation of $G$ (in $\left.G L_{n}\right)$ if the following conditions hold true:

- $S_{e}=T_{e}=\mathrm{id}$,

- $T_{g} S_{g} S_{h}=S_{g h} T_{g}$,

- $S_{g} T_{g} T_{h}=T_{h g} S_{g}$.

The birepresentation $(S, T)$ is called associative, if the following simultaneous relations are satisfied:

$$
S_{g} S_{h}=S_{g h}, \quad T_{g} T_{h}=T_{h g}, \quad S_{g} T_{h}=T_{h} S_{g}, \quad \forall g, h \in G
$$

In general, birepresentations need not be associative even for groups. 


\section{$5 \quad$ Generators and structure functions}

The generators of a birepresentation $(S, T)$ are defined as follows:

$$
\left.S_{j} \doteq \frac{\partial S_{g}}{\partial g^{j}}\right|_{g=e},\left.\quad T_{j} \doteq \frac{\partial T_{g}}{\partial g^{j}}\right|_{g=e}, \quad j=1, \ldots, r
$$

The auxiliary functions of $G$ are defined as

$$
\left.v_{j}^{n}(g) \doteq \frac{\partial(g h)^{n}}{\partial h^{j}}\right|_{h=e}, \quad n, j=1, \ldots, r
$$

The structure functions $c_{j k}^{n}(g)$ of $G$ are defined by the generalized Maurer-Cartan equations

$$
v_{j}^{n}(g) \frac{\partial v_{k}^{i}(g)}{\partial g^{n}}-v_{k}^{n}(g) \frac{\partial v_{j}^{i}(g)}{\partial g^{n}} \doteq c_{j k}^{n}(g) v_{n}^{i}(g)
$$

One can check the initial conditions

$$
c_{j k}^{n}(e)=c_{j k}^{n}
$$

The algebra with structure functions $c_{j k}^{n}(g)$ may be called the derivative of the tangent algebra $\Gamma$ of $G$. It follows from the Belousov theory [1] of derivative quasigroups and loops that the derivative of the tangent Mal'tsev algebra of $G$ is a Mal'tsev algebra as well. In a sense, the derivative Mal'tsev algebra is a deformation of $\Gamma$ with the deformation parameter $g \in G$.

\section{Generalized Lie equations (GLE)}

Define the derivative generators of $(S, T)$ as follows:

$$
S_{j}(g) \doteq T_{g} S_{j} T_{g}^{-1}, \quad T_{j}(g) \doteq S_{g}^{-1} T_{j} S_{g}
$$

By direct calculations, one can check that

$$
S_{j}(g)+T_{j}(g)=S_{j}+T_{j}
$$

In what follows, the commutator of linear operators $A, B$ is denoted as $[A, B] \doteq A B-B A$.

Theorem 1 (generalized Lie equations). Let $(S, T)$ be a differentiable birepresentation of an analytic Moufang loop $G$. Then the birepresentation matrices $S_{g}, T_{g}(g \in G)$ satisfy the system of simultaneus differential equations

$$
\begin{aligned}
v_{j}^{n}(g) \frac{\partial S_{g}}{\partial g^{n}} & =S_{g} \overbrace{T_{g} S_{j} T_{g}^{-1}}^{S_{j}(g)}=S_{g} S_{j}(g) \\
& =S_{g} S_{j}+\underbrace{\left[S_{g}, T_{j}\right]}_{\text {associator }} \\
v_{j}^{n}(g) \frac{\partial T_{g}}{\partial g^{n}} & =\overbrace{S_{g}^{-1} T_{j} S_{g}}^{T_{j}(g)} T_{g}=T_{j}(g) T_{g} \\
& =T_{j} T_{g}+\underbrace{\left[S_{j}, T_{g}\right]}_{\text {associator }} .
\end{aligned}
$$

Proof. Differentiate the defining relations of a birepresentation $(S, T)$ with respect to $g$ and take $g=e$. 


\section{Generalized Lie-Cartan theorem}

Theorem 2 (generalized Lie-Cartan theorem). The integrability conditions of GLE read as commutation relations

$$
\begin{aligned}
& {\left[S_{j}(g), S_{k}(g)\right]=c_{j k}^{n}(g) S_{n}(g)-2\left[S_{j}(g), T_{k}(g)\right]} \\
& {\left[T_{j}(g), T_{k}(g)\right]=-c_{j k}^{n}(g) T_{n}(g)-2\left[T_{j}(g), S_{k}(g)\right]}
\end{aligned}
$$

Proof. Differentiate the GLE and use

$$
\frac{\partial^{2} S_{g}}{\partial g^{j} \partial g^{k}}=\frac{\partial^{2} S_{g}}{\partial g^{k} \partial g^{j}}, \quad \frac{\partial^{2} T_{g}}{\partial g^{j} \partial g^{k}}=\frac{\partial^{2} T_{g}}{\partial g^{k} \partial g^{j}}
$$

Corollary 3. If $(S, T)$ is an associative birepresentation of a Lie group $G$, then one gets the well known Lie algebra commutation relations (Lie-Cartan theorem)

$$
\left[S_{j}, S_{k}\right]-c_{j k}^{n} S_{n}=\left[T_{j}, T_{k}\right]+c_{j k}^{n} T_{n}=\left[S_{j}, T_{k}\right]=0
$$

\section{Yamagutian and Yamaguti brackets}

Let $g \in G$ and $x, y \in T_{e}(G)$. Denote

$$
S_{x}(g) \doteq x^{j} S_{j}(g), \quad T_{x}(g) \doteq x^{j} T_{j}(g), \quad[x, y]_{g}^{i} \doteq c_{j k}^{i}(g) x^{j} y^{k}, \quad i=1, \ldots, r
$$

Define the Yamagutian $Y_{g}$ by

$$
3 Y_{g}(x ; y) \doteq\left[S_{x}(g), S_{y}(g)\right]+\left[S_{x}(g), T_{y}(g)\right]+\left[T_{x}(g), T_{y}(g)\right]
$$

By direct calulations one can check that the Yamagutian obeys the constraints

$$
\begin{aligned}
& Y_{g}(x ; y)+Y_{g}(y ; x)=0 \\
& Y_{g}\left([x, y]_{g} ; z\right)+Y_{g}\left([y, z]_{g} ; x\right)+Y_{g}\left([z, x]_{g} ; y\right)=0
\end{aligned}
$$

The Yamaguti brackets $[\cdot, \cdot, \cdot]_{g}$ are defined $[8,9]$ in $T_{e}(G)$ by

$$
\begin{aligned}
{[x, y, z]_{g} } & \doteq\left[x,[y, z]_{g}\right]_{g}-\left[y,[x, z]_{g}\right]_{g}+\left[[x, y]_{g}, z\right]_{g} \\
& =J_{g}(x, y, z)+2\left[[x, y]_{g}, z\right]_{g}
\end{aligned}
$$

\section{Closure of integrability conditions}

It turns out that the commutation relations for derivative generators of the birepresentation $(S, T)$ can be presented in a closed Lie algebra form.

The integrability conditions of GLE can be written as follows:

$$
\begin{aligned}
& {\left[S_{x}(g), S_{y}(g)\right]=2 Y_{g}(x ; y)+\frac{1}{3} S_{[x, y]_{g}}(g)+\frac{2}{3} T_{[x, y]_{g}}(g)} \\
& {\left[S_{x}(g), T_{y}(g)\right]=-Y_{g}(x ; y)+\frac{1}{3} S_{[x, y]_{g}}(g)-\frac{1}{3} T_{[x, y]_{g}}(g)} \\
& {\left[T_{x}(g), T_{y}(g)\right]=2 Y_{g}(x ; y)-\frac{2}{3} S_{[x, y]_{g}}(g)-\frac{1}{3} T_{[x, y]_{g}}(g)}
\end{aligned}
$$

One can prove the reductivity conditions [5]

$$
6\left[Y_{g}(x ; y), S_{z}(g)\right]=S_{[x, y, z]_{g}}(g)
$$




$$
6\left[Y_{g}(x ; y), T_{z}(g)\right]=T_{[x, y, z]_{g}}(g)
$$

By using the above reductivity conditions it can be shown by direct calculations that the Yamagutian obeys the Lie algebra

$$
6\left[Y_{g}(x ; y), Y_{g}(z ; w)\right]=Y_{g}\left([x, y, z]_{g} ; w\right)+Y_{g}\left(z ;[x, y, w]_{g}\right)
$$

Dimension of the Lie algebra $(1)-(6)$ does not exceed $2 r+r(r-1) / 2$. The Jacobi identities are guaranteed by the defining identities of the Lie [7] and general Lie triple systems [8, 9] associated with the derivative Mal'tsev algebra of $T_{e}(G)$ of $G$.

The commutation relations of form (1) - (6) are well known from the theory of alternative algebras [10]. By taking $g=e$, the commutation relations $(1)-(6)$ give the Lie algebra of the multiplication group of the birepresentation $(S, T)$ of $G$. For field theoretical applications see [6].

\section{Acknowledgment}

The research was in part supported by the Estonian Science Foundation, Grant 6912.

\section{References}

[1] V. D. Belousov. Foundations of the Theory of Quasiqroups and Loops. Nauka, Moscow, 1967 (in Russian).

[2] R. Moufang. Zur Struktur von Alternativkörpern. Math. Ann. B110 (1935), 416-430.

[3] H. Pflugfelder. Quasigroups and Loops: Introduction. Heldermann Verlag, Berlin, 1990.

[4] A. I. Mal'tsev. Analytical loops. Matem. Sbornik. 36 (1955), 569-576 (in Russian).

[5] E Paal. Continuous Moufang transformations. Acta Appl. Math. 50 (1998), 77-91.

[6] E. Paal. Note on Moufang-Noether currents. Czech. J. Phys. 56 (2006), 1257-1262.

[7] O. Loos. Über eine Beziehung zwischen Malcev-Algebren und Lie-Tripelsystemen. Pacific J. Math. 18 (1966), 553-562.

[8] K. Yamaguti. Note on Malcev algebras. Kumamoto J. Sci. A5 (1962), 203-207.

[9] K. Yamaguti. On the theory of Malcev algebras. Kumamoto J. Sci. A6 (1963), 9-45.

[10] R. D. Schafer. An Introduction to Nonassociative Algebras. Academic Press, New York, 1966. 